Bull. Korean Math. Soc. 48 (2011), No. 2, pp. 387-396

DOI 10.4134/BKMS.2011.48.2.387

\title{
ON THE INITIAL VALUES OF SOLUTIONS OF A GENERAL FUNCTIONAL EQUATION
}

\author{
Jae-Young Chung and Dohan Kim
}

\begin{abstract}
We consider a general functional equation with time variable which arises when we investigate regularity problems of some general functional equations. As a result we prove the regularity of the initial values of the solutions. Also as an application we prove the regularity of solutions of some classical functional equations and their distributional versions.
\end{abstract}

\section{Introduction}

In this paper, as a unified approach we investigate the initial values as $t, s \rightarrow$ $0^{+}$of the solutions $\Phi: \mathbb{R}^{2 n} \times(0, \infty)^{2} \rightarrow \mathbb{C}, F_{k}, G_{k}: \mathbb{R}^{n} \times(0, \infty) \rightarrow \mathbb{C}, k=$ $1,2, \ldots, m$, of the functional equation

$$
\Phi(x, y, t, s)=\sum_{k=1}^{m} F_{k}(x, t) G_{k}(y, s), \quad x, y \in \mathbb{R}^{n}, t, s>0,
$$

where $\Phi, F_{k}, G_{k}, k=1,2, \ldots, m$, are smooth functions of $x, y$ for each $t, s>0$ with the conditions $(2.1) \sim(2.4)$ in the next section.

The equation (1.1) arises when we study the functional equation of J. M. Speiser, H. J. Whitehouse and N. J. Berg [15]

$$
f(x+y)+g(x-y)=\sum_{k=1}^{m} f_{k}(x) g_{k}(y)
$$

in the space of distributions and hyperfunctions (see Section 3). The equation (1.2) has a significant application in signal processing. Furthermore, many important functional equations such as the Cauchy equations, exponential equations, quadratic functional equation, d'Alembert functional equation, trigonometric functional equations are special cases of the equation. The regularity of solutions of the functional equation (1.2) and its generalization are well known. As a matter of fact, under the natural assumption that $\left\{f_{1}, \ldots, f_{m}\right\}$

Received August 31, 2009.

2010 Mathematics Subject Classification. 39B82, 46F10.

Key words and phrases. functional equation, convolution, generalized function, heat kernel.

(C)2011 The Korean Mathematical Society 
and $\left\{g_{1}, \ldots, g_{m}\right\}$ are linearly independent, it was shown in [2] that the locally integrable solutions (which was weakened to Lebesgue measurability by Jarai [13]) $f, g, f_{k}, g_{k}: \mathbb{R}^{n} \rightarrow \mathbb{C}, k=1,2, \ldots, m$, of the equation (1.1) are smooth functions in which a usual convolutional technique was used. Convolving in (1.2) some regularizing functions in both variables $x$ and $y$ as in $[4,5,7]$, which is a different approach as in $[1,2]$, the equation (1.2) and its generalization in [2] are converted to the equations of the form (1.1).

As an application, we consider the regularity of the solutions of the following distributional version of the equation (1.1),

$$
u \circ L_{1}+v \circ L_{2}=\sum_{k=1}^{m} u_{k} \otimes v_{k},
$$

where $u, v, u_{k}, v_{k} \in \mathcal{G}^{\prime}\left(\mathbb{R}^{n}\right)$, and $\circ$ denotes the pullback, $\otimes$ denotes the tensor product of generalized functions, and $L_{1}(x, y)=x+y, L_{2}(x, y)=x-y$.

\section{Initial values of solutions of (1.1)}

We impose the following conditions on the functions $\Phi, F_{k}, G_{k}, k=1,2, \ldots, m$, in the equation (1.1): There exists $t_{1}>0$ such that

$$
\left\{F_{1}\left(\cdot, t_{1}\right), \ldots, F_{m}\left(\cdot, t_{1}\right)\right\}
$$

is linearly independent, and there exists $s_{1}>0$ such that

$$
\left\{G_{1}\left(\cdot, s_{1}\right), \ldots, G_{m}\left(\cdot, s_{1}\right)\right\}
$$

is linearly independent; for each fixed $y \in \mathbb{R}^{n}$ and $s>0$,

$$
\lim _{t \rightarrow 0^{+}} \Phi(x, y, t, s)
$$

is a smooth function of $x$, and for each fixed $x \in \mathbb{R}^{n}$ and $t>0$,

$$
\lim _{s \rightarrow 0^{+}} \Phi(x, y, t, s)
$$

is a smooth function of $y$.

From now on, we assume the condition $(2.1) \sim(2.4)$. Now we prove that the initial values

$$
\phi(x, y)=\lim _{t, s \rightarrow 0^{+}} \Phi(x, y, t, s), f_{k}(x)=\lim _{t \rightarrow 0^{+}} F_{k}(x, t), g_{k}(y)=\lim _{s \rightarrow 0^{+}} G_{k}(y, s) .
$$

of the solutions of the functional equation (1.1) are smooth functions for $k=$ $1,2, \ldots, m$.

Theorem 2.1. The initial values $\phi(x, y)$ and $f_{k}(x), g_{k}(y), k=1,2, \ldots, m$, of the solutions $\Phi, F_{k}, G_{k}, k=1,2, \ldots, m$, of the equation (1.1) smooth functions satisfying the functional equation

$$
\phi(x, y)=\sum_{k=1}^{m} f_{k}(x) g_{k}(y) .
$$


Proof. We first prove that $f_{k}(x)=\lim _{t \rightarrow 0^{+}} F_{k}(x, t)$ are smooth functions for all $k=1,2, \ldots, m$. We use the mathematical induction on $m$. For $m=1$, we can choose $y_{1} \in \mathbb{R}^{n}$ such that $G_{1}\left(y_{1}, s_{1}\right):=b_{1} \neq 0$ since $\left\{G_{1}\left(\cdot, s_{1}\right)\right\}$ is linear independent. Thus, it follows from the equation (1.1) and the condition (2.3) that

$$
f_{1}(x)=b_{1}^{-1} \lim _{t \rightarrow 0^{+}} \Phi_{1}\left(x, y_{1}, t, s_{1}\right)
$$

is a smooth function. Assume that the result holds for $m=p$, that is, $f_{1}, \ldots, f_{p}$ are smooth functions provided that the conditions (2.2) and (2.3) hold for $m=p$. We now prove that the result holds for $m=p+1$. Let

$$
\Phi(x, y, t, s)=\sum_{k=1}^{p+1} F_{k}(x, t) G_{k}(y, s)
$$

and assume that the conditions (2.2) and (2.3) hold for $m=p+1$. By the condition $(2.2)$, we may choose $y_{p+1} \in \mathbb{R}^{n}$ such that $G_{p+1}\left(y_{p+1}, s_{1}\right):=b_{p+1} \neq$ 0 . Then it follows from (2.8) that

$$
F_{p+1}(x, t)=b_{p+1}^{-1}\left(\Phi\left(x, y_{p+1}, t, s_{1}\right)-\sum_{k=1}^{p} b_{k} F_{k}(x, t)\right),
$$

where $b_{k}=G_{k}\left(y_{p+1}, s_{1}\right), k=1, \ldots, p$. Putting (2.9) in (2.8) we have

$$
\Phi^{*}(x, y, t, s)=\sum_{k=1}^{p} F_{k}(x, t) G_{k}^{*}(y, s)
$$

where

$$
\begin{aligned}
& \Phi^{*}(x, y, t, s)=\Phi(x, y, t, s)-b_{p+1}^{-1} \Phi\left(x, y_{p+1}, t, s_{1}\right) G_{p+1}(y, s), \\
& G_{k}^{*}(y, s)=G_{k}(y, s)-b_{p+1}^{-1} b_{k} G_{p+1}(y, s), k=1, \ldots, p .
\end{aligned}
$$

In view of (2.11) and (2.12) it is easy to see that $\Phi^{*}$ and $G_{k}^{*}, k=1,2, \ldots, p$, satisfy the conditions (2.2) and (2.3), respectively. Thus, by the induction hypothesis that the initial values

$$
f_{k}(x)=\lim _{t \rightarrow 0^{+}} F_{k}(x, t), k=1,2, \ldots, p
$$

are smooth functions, it follows from (2.9) that

$$
f_{p+1}(x):=\lim _{t \rightarrow 0^{+}} F_{p+1}(x, t)=b_{p+1}^{-1} \lim _{t \rightarrow 0^{+}} \Phi^{*}\left(x, y_{p+1}, t, s_{1}\right)-b_{p+1}^{-1} \sum_{k=1}^{p} b_{k} f_{k}(x)
$$

is a smooth function since $\Phi^{*}$ satisfies $(2.3)$.

Changing the roles of $F_{k}$ and $G_{k}$ for $k=1,2, \ldots, m$, we obtain, for each $k=1,2, \ldots, m$,

$$
g_{k}(y):=\lim _{t \rightarrow 0^{+}} G_{k}(y, t)
$$


is a smooth function. Finally, letting $t, s \rightarrow 0^{+}$in (1.1) we get (2.6). This completes the proof.

If the functions $\Phi, F_{k}, G_{k}, k=1,2, \ldots, m$, in Theorem 1.1 are independent of the parameters $t$ and $s$, Theorem 1.2 can be stated as follows.

Corollary 2.2. Let $\Phi(x, y)$ be a smooth function of each variable, and let $\left\{F_{1}, \ldots, F_{m}\right\}$ and $\left\{G_{1}, \ldots, G_{m}\right\}$ be linearly independent. Then the solutions $F_{k}, G_{k}, k=1,2, \ldots, m$, of the equation

$$
\Phi(x, y)=\sum_{k=1}^{m} F_{k}(x) G_{k}(y)
$$

are smooth functions.

Using the above results we obtain the following result in [2].

Theorem 2.3. The locally integrable solutions $f, g, f_{k}, g_{k}: \mathbb{R}^{n} \rightarrow \mathbb{C}, k=$ $1,2 \ldots, m$, of the equation

$$
f(x+y)+g(x-y)=\sum_{k=1}^{m} f_{k}(x) g_{k}(y)
$$

are smooth functions under the natural assumptions that $\left\{f_{1}, \ldots, f_{m}\right\}$ and $\left\{g_{1}, \ldots, g_{m}\right\}$ are linearly independent.

Proof. We choose a smooth function $\psi$ on $\mathbb{R}^{n}$ such that

$$
\begin{aligned}
& \psi(x) \geq 0 \text { for all } x \in \mathbb{R}^{n}, \\
& \operatorname{supp} \psi \subset\left\{x \in \mathbb{R}^{n}:|x| \leq 1\right\}, \\
& \int_{\mathbb{R}^{n}} \psi(x) d x=1
\end{aligned}
$$

and let

$$
\Psi(x, y, t, s):=t^{-n} s^{-n} \psi\left(\frac{x}{t}\right) \psi\left(\frac{y}{s}\right), t, s>0 .
$$

Convolving $\Psi(x, y, t, s)$ in both sides of (2.15) as a function of $x$ and $y$ we have

$$
\left(f * \psi_{t} * \psi_{s}\right)(x+y)+\left(g * \psi_{t} * \psi_{s}\right)(x-y)=\sum_{k=1}^{m}\left(f_{k} * \psi_{t}\right)(x)\left(g_{k} * \psi_{s}\right)(y)
$$

Now we let

$$
\begin{aligned}
\Phi(x, y, t, s) & =\left(f * \psi_{t} * \psi_{s}\right)(x+y)+\left(g * \psi_{t} * \psi_{s}\right)(x-y), \\
F_{k}(x, t) & =\left(f_{k} * \psi_{t}\right)(x), \\
G_{k}(y, s) & =\left(g_{k} * \psi_{s}\right)(y) .
\end{aligned}
$$

Then it is easy to see that $\Phi, F_{k}, G_{k}, k=1,2 \ldots, m$, satisfy the conditions $(2.1) \sim(2.4)$. Thus it follows from Theorem 2.1 that the initial values

$$
\tilde{f}_{k}(x):=\lim _{t \rightarrow 0^{+}} F_{k}(x), \quad \widetilde{g}_{k}(x):=\lim _{s \rightarrow 0^{+}} G_{k}(y), \widetilde{\phi}(x, y):=\lim _{t, s \rightarrow 0^{+}} \Phi(x, y, t, s)
$$


are all smooth functions. Now, replacing $x+y$ by $u, x-y$ by $v$ and letting $t, s \rightarrow 0^{+}$in $(2.17)$ we have

$$
\widetilde{\phi}\left(\frac{u+v}{2}, \frac{u-v}{2}\right)=\widetilde{f}(u)+g(v)
$$

for all $u \in \mathbb{R}^{n}$ and for all $v \in \mathbb{R}^{n}$ such that $\lim _{t \rightarrow 0^{+}}\left(g * \psi_{t}\right)(v)=g(v)$, where

$$
\tilde{f}(u)=\lim _{t, s \rightarrow 0^{+}}\left(f * \psi_{t} * \psi_{s}\right)(u) \text {. }
$$

Thus, in view of $(2.20), \widetilde{f}$ is a smooth function and so is $\widetilde{g}(x):=\lim _{t, s \rightarrow 0^{+}}(g *$ $\left.\psi_{t} * \psi_{s}\right)(x)$. Letting $t, s \rightarrow 0^{+}$in $(2.16)$ we have

$$
\widetilde{f}(x+y)+\widetilde{g}(x-y)=\sum_{k=1}^{m} \widetilde{f}_{k}(x) \widetilde{g}_{k}(y)
$$

Note that there exists $\Omega \subset \mathbb{R}^{n}$ with the Lebesgue measure $m\left(\Omega^{c}\right)=0$ such that

$$
\widetilde{f}(x)=f(x), \widetilde{g}(x)=g(x), \widetilde{f}_{k}(x)=f_{k}(x), \widetilde{g}_{k}(x)=g_{k}(x)
$$

for all $x \in \Omega$ and $k=1,2, \ldots, m$. For given $z \in \mathbb{R}^{n}$, choose $\omega \in(z-2 \Omega) \cap(2 \Omega-$ $z) \cap \Omega$ and put $x=\frac{1}{2}(z+\omega), y=\frac{1}{2}(z-\omega)$, where $z-2 \Omega=\{z-2 \omega: \omega \in \Omega\}$, $2 \Omega-z=\{2 \omega-z: \omega \in \Omega\}$. Then we have

$$
\widetilde{f}(z)+g(x-y)=\sum_{k=1}^{m} f_{k}(x) g_{k}(y)
$$

since $x, y, x-y \in \Omega$. In view of (2.15) and (2.22) we have $\widetilde{f}(x)=f(x)$ for all $x \in \mathbb{R}^{n}$. Similarly we can show that $\widetilde{g}(x)=g(x)$ for all $x \in \mathbb{R}^{n}$. Thus we have proved that $f$ and $g$ are smooth functions. Finally, applying Corollary 2.2 we obtain that $f_{k}, g_{k}, k=1,2, \ldots, m$ are smooth functions. This completes the proof.

Remark. As a generalization of the equation (1.2), Aczél and J. K. Chung introduced the following functional equation [2]

$$
\sum_{j=1}^{l} h_{j}\left(a_{j} x+b_{j} y\right)=\sum_{k=1}^{m} f_{k}(x) g_{k}(y)
$$

where $h_{j}, f_{k}, g_{k}: \mathbb{R} \rightarrow \mathbb{C}, a_{j}, b_{j} \in \mathbb{R}, a_{j} b_{j} \neq 0, a_{i} b_{j} \neq a_{j} b_{i}$ for all $i \neq j, i, j=$ $1, \ldots, l, k=1, \ldots, m$, and $\left\{g_{1}, \ldots, g_{m}\right\}$ and $\left\{h_{1}, \ldots, h_{m}\right\}$ are linearly independent.

Using the same approach as in the proof of Theorem 2.3, we obtain the equation

$$
\sum_{j=1}^{l}\left(h_{j} * \psi_{t, a_{j}} * \psi_{s, b_{j}}\right)\left(a_{j} x+b_{j} y\right)=\sum_{k=1}^{m}\left(f_{k} * \psi_{t}\right)(x)\left(g_{k} * \psi_{s}\right)(y)
$$


instead of $(2,16)$, and the equation $(2.17)$ is replaced by

$$
\Phi(x, y, t, s)=\sum_{j=1}^{l}\left(h_{j} * \psi_{t, a_{j}} * \psi_{s, b_{j}}\right)\left(a_{j} x+b_{j} y\right),
$$

where $\psi_{t, a}(x)=a^{-1} \psi_{t}\left(a^{-1} x\right)$ for $a=a_{j}, b_{j}, j=1,2, \ldots, l$. Now, as in [5], letting $s \rightarrow 0^{+}$in $(2.25)$, replacing $x$ by $a_{i}^{-1}\left(x-b_{i} y\right)$, multiplying $\psi_{s}(y)$, integrating with respect to $y$, and letting $t \rightarrow 0^{+}$we have

$$
\widetilde{h}_{i}(x):=-\sum_{j \neq i}\left(h_{j} * \psi_{s, c_{j}}\right)(x)+\sum_{k=1}^{m} \int \widetilde{\phi}\left(a_{i}^{-1} x-a_{i}^{-1} b_{i} y, y\right) \psi_{s}(y) d y
$$

where $c_{j}=a_{i}^{-1}\left(b_{i} a_{j}-a_{i} b_{j}\right)$ for all $1 \leq j \leq l, j \neq i, \widetilde{h}_{j}(x)=\lim _{t \rightarrow 0^{+}}\left(h_{j} *\right.$ $\left.\psi_{t, a_{j}}\right)(u)$ and $\widetilde{\phi}$ is defined as in Theorem 2.3. Thus $\widetilde{h}_{j}$ is smooth function for each $j=1,2, \ldots, l$. Letting $t, s \rightarrow 0^{+}$in $(2.24)$ we have

$$
\sum_{j=1}^{l} \widetilde{h}_{j}\left(a_{j} x+b_{j} y\right)=\sum_{k=1}^{m} \widetilde{f}_{k}(x) \widetilde{g}_{k}(y)
$$

Let $\Omega \subset \mathbb{R}$ such that Lebesgue measure $m\left(\Omega^{c}\right)=0$ and for all $x \in \Omega$,

$\widetilde{f}_{k}(x)=f_{k}(x), \widetilde{g}_{k}(x)=g_{k}(x), \widetilde{h}_{j}(x)=h_{j}(x), k=1,2, \ldots, m, j=1,2, \ldots, l$.

For each fixed $i, 1 \leq i \leq l$, and $z \in \mathbb{R}$, choose

$$
\omega \in \bigcap_{1 \leq j \leq l, j \neq i}\left[\frac{2 a_{i} b_{i}}{a_{j} b_{i}-a_{i} b_{j}} \Omega-\left(\frac{a_{j} b_{i}+a_{i} b_{j}}{a_{j} b_{i}-a_{i} b_{j}}\right) z\right] \cap \Omega
$$

and put in $(2.23)$,

$$
x=\frac{1}{2 a_{i}}(z+\omega), \quad y=\frac{1}{2 b_{i}}(z-\omega),
$$

where for $\alpha, \beta \in \mathbb{R}$ we denote by $\alpha \Omega+\beta=\{\alpha \omega+\beta: \omega \in \Omega\}$. Then it is obvious that $a_{i} x+b_{i} y=z$ and $x, y, a_{j} x+b_{j} y \in \Omega$ for all $1 \leq j \leq l, j \neq i$. Thus we have

$$
h_{i}(z)+\sum_{j \neq i} \widetilde{h}_{j}\left(a_{j} x+b_{j} y\right)=\sum_{k=1}^{m} \widetilde{f}_{k}(x) \widetilde{g}_{k}(y) .
$$

In view of (2.23) and (2.28), we have $h_{i}=\tilde{h}_{i}$. Thus $h_{j}$ are smooth functions for all $j=1,2, \ldots, l$. Thus we have the following result.

Theorem 2.4. The locally integrable solutions $h_{j}, g, f_{k}, g_{k}: \mathbb{R}^{n} \rightarrow \mathbb{C}, j=$ $1,2, \ldots, l, k=1,2 \ldots, m$, of the equation (2.23) are smooth functions under the conditions that $\left\{f_{1}, \ldots, f_{m}\right\}$ and $\left\{g_{1}, \ldots, g_{m}\right\}$ are linearly independent.

Examples. The following functional equations are typical examples of the equation (1.2).

$$
f(x+y)+f(x-y)-2 f(x)-2 g(y)=0,
$$




$$
\begin{aligned}
& f(x+y)+f(x-y)-2 f(x) f(y)=0, \\
& f(x+y)+f(x-y)-2 f(x) g(y)=0, \\
& f\left(\frac{x+y}{2}\right)-f\left(\frac{x-y}{2}\right)-g(x) g(y)=0, \\
& f(x-y)-f(x) f(y)-g(x) g(y)=0,
\end{aligned}
$$

where $f, g: \mathbb{R}^{n} \rightarrow \mathbb{C}$. As a consequence of our result the Lebesgue integrable solutions of the equations $(2.29) \sim(2.33)$ are smooth functions. Now, it can be verified that the smooth solutions $f, g$ of the equations $(2.29) \sim(2.33)$ are given, respectively, by

$$
\begin{aligned}
g(x) & =\sum_{1 \leq j \leq k \leq n} a_{j k} x_{j} x_{k}, \\
f(x) & =\sum_{1 \leq j \leq k \leq n} a_{j k} x_{j} x_{k}+\sum_{j=1}^{n} b_{j} x_{j}+d, \\
f(x) & =\cos \left(a_{1} x_{1}+\cdots+a_{n} x_{n}\right), \\
g(x) & =\cos \left(a_{1} x_{1}+\cdots+a_{n} x_{n}\right), \\
f(x) & =c_{1} \cos \left(a_{1} x_{1}+\cdots+a_{n} x_{n}\right)+c_{2} \sin \left(a_{1} x_{1}+\cdots+a_{n} x_{n}\right), \\
g(x) & =c \sin \left(a_{1} x_{1}+\cdots+a_{n} x_{n}\right), \\
f(x) & =c^{2} \sin ^{2}\left(a_{1} x_{1}+\cdots+a_{n} x_{n}\right)+d,
\end{aligned}
$$

or

$$
\begin{aligned}
& g(x)=a_{1} x_{1}+\cdots+a_{n} x_{n}, \\
& f(x)=\left(a_{1} x_{1}+\cdots+a_{n} x_{n}\right)^{2}+d, \\
& g(x)=\sin \left(a_{1} x_{1}+\cdots+a_{n} x_{n}\right), \\
& f(x)=\cos \left(a_{1} x_{1}+\cdots+a_{n} x_{n}\right),
\end{aligned}
$$

where $x=\left(x_{1}, \ldots, x_{n}\right)$ and all the coefficients are complex numbers.

\section{Functional equations in Gelfand-Shilov generalized functions}

We briefly introduce the space $\mathcal{G}\left(\mathbb{R}^{n}\right)$ of generalized function of Gelfand and Shilov. Here we use the following notations: $|x|=\sqrt{x_{1}^{2}+\ldots+x_{n}^{2}},|\alpha|=$ $\alpha_{1}+\cdots+\alpha_{n}, \alpha !=\alpha_{1} ! \cdots \alpha_{n} !, x^{\alpha}=x_{1}^{\alpha_{1}} \cdots x_{n}^{a_{n}}$ and $\partial^{\alpha}=\partial_{1}^{\alpha_{1}} \cdots \partial_{n}^{\alpha_{n}}$ for $x=\left(x_{1}, \ldots, x_{n}\right) \in \mathbb{R}^{n}, \alpha=\left(\alpha_{1}, \ldots, \alpha_{n}\right) \in \mathbb{N}_{0}^{n}$, where $\mathbb{N}_{0}$ is the set of nonnegative integers and $\partial_{j}=\frac{\partial}{\partial x_{j}}$.

Definition 3.1 ([11]). We denote by $\mathcal{G}$ or $\mathcal{G}\left(\mathbb{R}^{n}\right)$ the Gelfand space of all infinitely differentiable functions $\varphi$ in $\mathbb{R}^{n}$ such that

$$
\|\varphi\|_{h, k}=\sup _{x \in \mathbb{R}^{n}, \alpha, \beta \in \mathbb{N}_{0}^{n}} \frac{\left|x^{\alpha} \partial^{\beta} \varphi(x)\right|}{h^{|\alpha|} k^{|\beta|} \alpha !^{1 / 2} \beta !^{1 / 2}}<\infty
$$


for some $h, k>0$. We say that $\varphi_{j} \longrightarrow 0$ as $j \rightarrow \infty$ if $\left\|\varphi_{j}\right\|_{h, k} \longrightarrow 0$ as $j \rightarrow \infty$ for some $h, k$, and denote by $\mathcal{G}^{\prime}$ the dual space of $\mathcal{G}$ and call its elements Gelfand generalized functions.

It is known that the space $\mathcal{G}\left(\mathbb{R}^{n}\right)$ consists of all infinitely differentiable functions $\varphi(x)$ on $\mathbb{R}^{n}$ which can be extended to an entire function on $\mathbb{C}^{n}$ satisfying

$$
|\varphi(x+i y)| \leq C \exp \left(-a|x|^{2}+b|y|^{2}\right)
$$

for some $a, b, C>0$ (see [11]). In view of (3.1) it is easy to see that the $n$-dimensional heat kernel $E_{t}(x)$ given by

$$
E_{t}(x)=(4 \pi t)^{-n / 2} \exp \left(-|x|^{2} / 4 t\right), t>0
$$

belongs to the Gelfand space $\mathcal{G}\left(\mathbb{R}^{n}\right)$ for each $t>0$.

We briefly introduce some basic operations on the spaces of the generalized functions.

Definition 3.2. Let $u_{j} \in \mathcal{G}^{\prime}\left(\mathbb{R}^{n_{j}}\right)$ for $j=1,2$, with $n_{1} \geq n_{2}$, and let $\lambda$ : $\mathbb{R}^{n_{1}} \rightarrow \mathbb{R}^{n_{2}}$ be a smooth function such that for each $x \in \mathbb{R}^{n_{1}}$, the Jacobian matrix $\nabla \lambda(x)$ of $\lambda$ at $x$ has rank $n_{2}$. Then there exists a unique continuous linear map $\lambda^{*}: \mathcal{G}^{\prime}\left(\mathbb{R}^{n_{2}}\right) \rightarrow \mathcal{G}^{\prime}\left(\mathbb{R}^{n_{1}}\right)$ such that $\Lambda^{*} u=u \circ \lambda$ when $u$ is a continuous function. We call $\lambda^{*} u$ the pullback of $u$ by $\lambda$ and often denoted by $u \circ \lambda$.

In particular if $\lambda$ is a diffeomorphism (a bijection with $\lambda, \lambda^{-1}$ smooth functions) the pullback $u \circ \lambda$ can be written as follows:

$$
\langle u \circ \lambda, \varphi\rangle=\left\langle u,\left(\varphi \circ \lambda^{-1}\right)(x)\left|\nabla \lambda^{-1}(x)\right|\right\rangle .
$$

Definition 3.3. Let $u_{j} \in \mathcal{G}^{\prime}\left(\mathbb{R}^{n_{j}}\right), j=1,2$. Then the tensor product $u_{1} \otimes u_{2}$ of $u_{1}$ and $u_{2}$ is defined by

$$
\left\langle u_{1} \otimes u_{2}, \varphi\left(x_{1}, x_{2}\right)\right\rangle=\left\langle u_{1},\left\langle u_{2}, \varphi\left(x_{1}, x_{2}\right)\right\rangle\right\rangle, \quad \varphi\left(x_{1}, x_{2}\right) \in C_{c}^{\infty}\left(\mathbb{R}^{n_{1}} \times \mathbb{R}^{n_{2}}\right) .
$$

The tensor product $u_{1} \otimes u_{2}$ belongs to $\mathcal{D}^{\prime}\left(\mathbb{R}^{n_{1}} \times \mathbb{R}^{n_{2}}\right)$.

For more details of pullbacks and tensor products of distributions we refer the reader to Chapter VI of [12].

Now we consider the functional equation

$$
u \circ L_{1}+v \circ L_{2}=\sum_{k=1}^{m} u_{k} \otimes v_{k},
$$

where $u, v, u_{k}, v_{k} \in \mathcal{G}\left(\mathbb{R}^{n}\right)$, and $\circ$ denotes the pullback, $\otimes$ denotes the tensor product of generalized functions, and $L_{1}(x, y)=x+y, L_{2}(x, y)=x-y$.

Theorem 3.4. The solutions $u, v, u_{k}, v_{k} \in \mathcal{G}\left(\mathbb{R}^{n}\right), k=1, \ldots, m$, of the equation (3.3) are smooth functions satisfying the classical functional equation (1.1). 
Proof. We employ the heat kernel $E_{t}$ defined by

$$
E_{t}(x)=(4 \pi t)^{-n / 2} \exp \left(-|x|^{2} / 4 t\right), \quad t>0 .
$$

Let $u \in \mathcal{G}\left(\mathbb{R}^{n}\right)$. Then, for each $t>0,\left(u * E_{t}\right)(x):=\left\langle u_{y}, E_{t}(x-y)\right\rangle$ is a smooth function of $x$. Convolving the tensor product $E_{t}(x) E_{s}(y)$ in both sides of (3.1) we have

$$
\begin{aligned}
{\left[\left(u \circ L_{1}\right) *\left(E_{t}(x) E_{s}(y)\right)\right](\xi, \eta) } & =\left\langle u \circ L_{1}, E_{t}(\xi-x) E_{s}(\eta-y)\right\rangle \\
& =\left\langle u, \int E_{t}(\xi-x+y) E_{s}(\eta-y) d y\right\rangle \\
& =\left\langle u,\left(E_{t} * E_{s}\right)(\xi+\eta-x)\right\rangle \\
& =\left\langle u, E_{t+s}(\xi+\eta-x)\right\rangle \\
& =\left(u * E_{t+s}\right)(\xi+\eta) .
\end{aligned}
$$

Similarly we have

$$
\left[\left(v \circ L_{2}\right) *\left(E_{t}(x) E_{s}(y)\right)\right](\xi, \eta)=\left(v * E_{t+s}\right)(\xi-\eta),
$$

and

$$
\left[\left(u_{k} \otimes v_{k}\right) *\left(E_{t}(x) E_{s}(y)\right)\right](\xi, \eta)=\left(u_{k} * E_{t}\right)(\xi)\left(v_{k} * E_{s}\right)(\eta)
$$

for all $k=1, \ldots, m$. Thus the equation (3.1) is converted to the functional equation of the form

$$
\Phi(x, y, t, s)=\sum_{k=1}^{m} U_{k}(x, t) V_{k}(y, s),
$$

where

$$
\begin{aligned}
\Phi(x, y, t, s) & =\left(u * E_{t+s}\right)(x+y)+\left(v * E_{t+s}\right)(x-y), \\
U_{k}(x, t) & =\left(u_{k} * E_{t}\right)(x), \\
V_{k}(x, t) & =\left(v_{k} * E_{s}\right)(y)
\end{aligned}
$$

for all $k=1,2, \ldots, m$. Applying Theorem 2.1 we obtain the result.

Combined with the result of Aczél and Chung [2] we have the following corollary as a consequence of the above result.

Corollary 3.5. Every solution $u, v, u_{k}, v_{k} \in \mathcal{G}(\mathbb{R}), k=1, \ldots, m$, of the equation (3.3) has the form of exponential polynomials

$$
\sum_{k=1}^{q} e^{r_{k} x} p_{k}(x)
$$

where $r_{k} \in \mathbb{C}$ and $p_{k}{ }^{\prime} s$ are polynomials for all $k=1,2, \ldots, q$.

Acknowledgment. The authors express their sincere gratitude to the referee for the useful comments on the paper. The first author was supported by the Korea Research Foundation Grant(KRF) grant funded by the Korea 
Government(MEST)(No. 2009-0063887) and the second author was partially supported by the Research Institute of Mathematics, Seoul National University.

\section{References}

[1] J. Aczél and J. Dhombres, Functional Equations in Several Variables, Cambridge University Press, Cambridge, 1989.

[2] J. Aczél and J. K. Chung, Integrable solutions of functional equations of a general type, Studia Sci. Math. Hungar. 17 (1982), no. 1-4, 51-67.

[3] J. Chang and J. Chung, The stability of the sine and cosine functional equations in Schwartz distributions, Bull. Korean Math. Soc. 46 (2009), no. 1, 87-97.

[4] J. Chung, A functional equation of Aczél and Chung in generalized functions, Adv. Difference Equ. 2008 (2008), Art. ID 147979, 11 pp.

[5] _ Stability of approximately quadratic Schwartz distributions, Nonlinear Anal. 67 (2007), no. 1, 175-186.

[6] _ A distributional version of functional equations and their stabilities, Nonlinear Anal. 62 (2005), no. 6, 1037-1051.

7] J. Chung, S.-Y. Chung, and D. Kim, Generalized Pompeiu equation in distributions, Appl. Math. Lett. 19 (2006), no. 5, 485-490.

[8] E. Y. Deeba, E. L. Koh, P. K. Sahoo, and S. Xie, On a distributional analog of a sum form functional equation, Acta Math. Hungar. 78 (1998), no. 4, 333-344.

[9] E. Deeba, P. K. Sahoo, and S. Xie, On a class of functional equations in distribution, J. Math. Anal. Appl. 223 (1998), no. 1, 334-346.

[10] E. Y. Deeba and S. Xie, Distributional analog of a functional equation, Appl. Math. Lett. 16 (2003), no. 5, 669-673.

[11] I. M. Gelfand and G. E. Shilov, Generalized Functions. II, Academic Press, New York, 1968.

[12] L. Hörmander, The Analysis of Linear Partial Differential Operators. I, Springer-Verlag, Berlin-New York, 1983.

[13] A. Jarai, A remark to a paper of J. Aczél and J. K. Chung, Studia Sci. Math. Hungar. 19 (1984), no. 2-4, 273-274.

[14] L. Schwartz, Théorie des distributions, Hermann, Paris, 1966.

[15] J. M. Speiser, H. J. Whitehouse, and N. J. Berg, Signal processing architectures using convolutional technology, Real time Signal Processing, SPIE 154 (1978), 66-80.

[16] L. Székelyhidi, The stability of the sine and cosine functional equations, Proc. Amer. Math. Soc. 110 (1990), no. 1, 109-115.

[17] _ On the Levi-Civita Functional Equation, Forschungszentrum Graz, Mathematisch-Statistische Sektion, Graz, 1988.

JAe-Young Chung

Department of Mathematics

Kunsan NATIONAL University

Kunsan 573-701, KoreA

E-mail address: jychung@kunsan.ac.kr

DOHAN KIM

Department of Mathematics

Seoul National University

SEOUL 151-747, KorEA

E-mail address: dhkim@math.snu.ac.kr 